\title{
eJRIEPS
}

Ejournal de la recherche sur l'intervention en éducation physique et sport

Hors-série $N^{\circ} 2 \mid 2018$

À propos de certaines bases théoriques et pratiques des sports collectifs

\section{Analyser une situation de jeu : mode d'emploi}

Jean-Francis Gréhaigne

\section{OpenEdition}

Journals

Édition électronique

URL : http://journals.openedition.org/ejrieps/515

DOI : 10.4000/ejrieps.515

ISSN : 2105-0821

Éditeur

ELLIADD

\section{Référence électronique}

Jean-Francis Gréhaigne, "Analyser une situation de jeu : mode d'emploi », eJRIEPS [En ligne], Horssérie $N^{\circ} 2$ | 2018, mis en ligne le 01 juillet 2018, consulté le 01 août 2019. URL : http:// journals.openedition.org/ejrieps/515; DOI : 10.4000/ejrieps.515

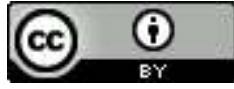

La revue eJRIEPS est mise à disposition selon les termes de la Creative Commons Attribution 4.0 International License. 


\section{Analyser une situation de jeu : mode d'emploi}

Mérand (1977) présente les jeux sportifs collectifs comme «une dimension spécifique, une modalité originale d'exploitation de la dynamique des groupes restreints poursuivant un objectif commun » (p. 12). Les joueurs doivent saisir comment s'organisent dialectiquement les actions individuelles et collectives en vue de réaliser une action commune.

Bien analyser une situation de jeux devient primordiale, mais n'est pas toujours très facile. Pourtant, cela a des conséquences sur les décisions prises en vue de construire des situations d'apprentissage. Aussi, nous allons revenir dans ce texte sur quelques critères qui permettent de mieux comprendre et donc d'analyser une situation de jeu en train de se dérouler. Une véritable activité pour les joueurs en sportifs collectifs consiste à observer, extraire et évaluer les informations pertinentes dans des situations de jeu permettant de réduire le temps d'analyse et donc de décision. En fonction du degré d'adéquation des réponses par rapport à la situation d'affrontement, il y a :

- soit proposition de modification des choix effectués en vue d'obtenir les effets attendus ;

- soit stabilisation des réponses par répétition ;

- soit complexification des apprentissages en vue d'amener l'élève à un niveau supérieur de

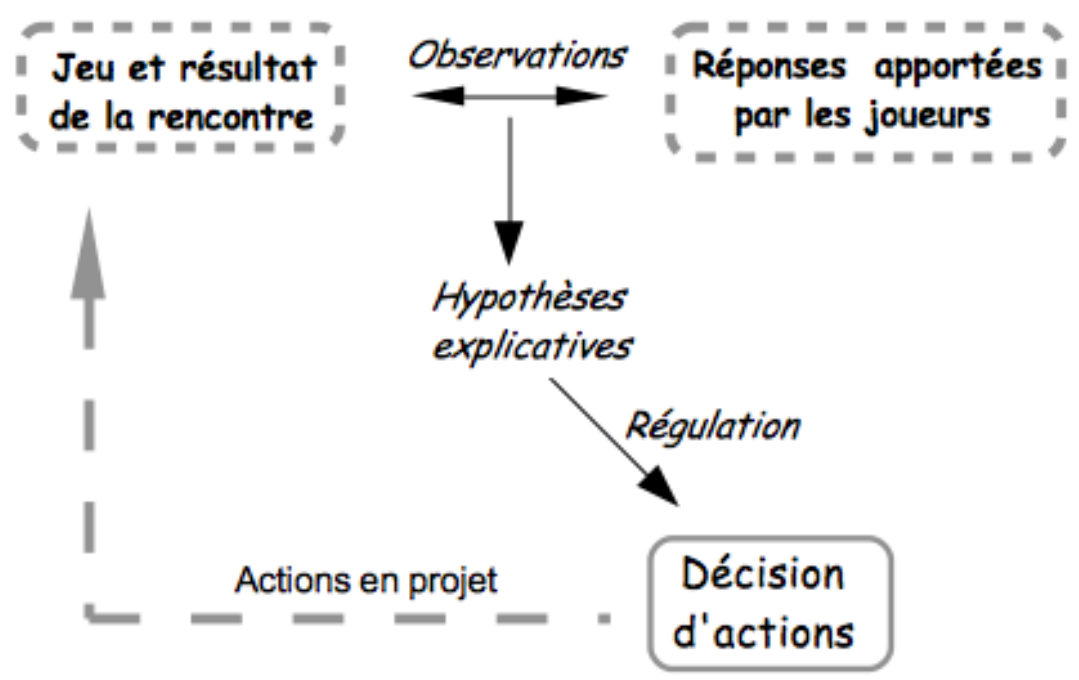

pratique.

Figure 1. Schéma d'aide à la décision pour les élèves et les enseignants.

Il s'agit pour les élèves de construire des connaissances et des compétences motrices par une succession d'hypothèses et d'essais. Cette construction s'appuyant sur une analyse des conditions ayant conduit à la réussite ou à l'échec. Autrement dit, à côté de l'action qui se satisfait de la notion 
de réussite (le but est atteint ou non), nous proposons, comme éléments indispensables à la formation en sport collectif, la prise de conscience des conditions de la réussite et mieux encore, la formalisation qui, seule, permet l'interprétation et l'explication de l'action. Le débat d'idées entre les élèves, via le guidage de l'enseignant, ou les propositions du professeur, renvoient à plusieurs types de situations d'apprentissage qui peuvent être proposées par le responsable dans ce type de travail.

- Des situations authentiques de jeu aménagé permettant d'affiner les compétences motrices et de régler les problèmes de timing. Par exemple en 3 x 3, jeu long obligatoire ou bien la situation d'apprentissage débute à la récupération du ballon.

- Des situations proches du jeu réel permettant de faire émerger les conditions stratégiques et tactiques de la réussite ; par exemple 4 x 4 sur terrain de $40 \mathrm{~m}$ sur $20 \mathrm{~m}$, avec des cibles de 3 x 2 sans gardien de but et des consignes facilitantes qui induisent des réponses.

- Des situations respectant la systémique du jeu permettant aux élèves de connaître, puis reconnaître, des configurations de jeu ; par exemple un jeu complet sur grand terrain ou 5 x 5 sur terrain de $50 \mathrm{~m}$ sur $30 \mathrm{~m}$, avec des cibles de $6 \times 2$ et gardien de but.

Bien entendu, nous raisonnons en termes de dominantes mais dans la pratique, les différentes situations d'apprentissage ne sont pas aussi nettement différenciées que cet exposé pourrait le laisser apparaître. Les apprentissages conscients ainsi réalisés permettent d'aboutir à la construction de compétences motrices qui ne sont pas isolées du contexte. La méthodologie employée attire l'attention de l'élève sur le repérage des conditions du faire et des conditions de réussite. De ce fait, la procédure devrait pouvoir être ré-investissable dans d'autres situations d'apprentissage.

Néanmoins, il faut avoir en tête qu'une situation d'apprentissage doit avoir une existence assez longue pour qu'elle soit efficace. Elle doit être présentée plusieurs fois aux élèves le temps que les processus d'appropriation et d'apprentissage puissent s'effectuer. Dans la conception des situations, un autre aspect qu'il nous paraît important de développer consiste à envisager le moment où le ballon est récupéré par la défense comme véritable point de départ de la situation jouée, qui devient par là même l'attaque. On est confronté de la sorte à des modalités qui sont très proches des véritables conditions du jeu. L'apprentissage réside dans la gestion et l'exécution du deuxième temps de jeu, préservant ainsi un minimum de désordre très utile quand on se retrouve face au fonctionnement réel du jeu.

\section{Situation d'apprentissage}

$\mathrm{Au}$ plan général, une situation didactique ou une série de situations doivent présenter des opportunités d'action et devrait posséder un potentiel d'évolution qui est à la disposition des élèves. Ceux-ci sont tenus d'identifier les facteurs favorables présents afin de transformer leurs connaissances et compétences. 
Un cycle de travail débute habituellement par une situation de référence qui permet une évaluation formative de départ des élèves (Cardinet, 1984 ; Gréhaigne, 2003). Il se poursuit avec des situations d'apprentissage sur lesquelles nous allons revenir. Et enfin, il se termine par une leçon où l'évaluation sommative tient une grande place. Une situation d'apprentissage se focalise sur l'émergence et la construction d'une ou plusieurs règles d'action (sur des connaissances) en relation avec les compétences motrices correspondantes. Dans une perspective socio-constructiviste de l'apprentissage, et pour aider les élèves à transformer et à augmenter leurs réponses disponibles dans différentes pratiques sociales de référence, le dispositif didactique doit comprendre :

- des temps d'action dans lesquels les élèves sont en activité motrice dans le jeu ;

- des temps d'observation pour permettre de prélever de l'information sur les actions ;

- des temps de « débats d'idées » (Deriaz, Poussin, \& Gréhaigne, 1998) afin qu'ils s'expriment et échangent sur les résultats et le processus de l'action en vue de transformer leurs actions.

La confrontation des élèves aux situations produit des centrations particulières tout au long des différentes périodes du processus d'apprentissage. Il y a d'abord une période d'appropriation et d'exploration dans laquelle les élèves sont appelés à circonscrire les exigences de la tâche et rechercher des effets positifs à partir des réponses dont ils disposent ou bien commencer à élaborer de nouvelles réponses. Puis, il y a une période de validation dans laquelle les élèves sont amenés à éprouver dans l'action la pertinence des actions retenues comme les plus efficientes pour atteindre le but de la tâche. Enfin, il y a une période d'intégration et de généralisation dans laquelle ils vont devoir exploiter et intégrer leurs apprentissages dans de nouvelles situations ou des situations plus complexes. Donc dans ce processus, il faut prendre son temps et bien différencier ce qui relève du simple changement, avec l'émergence puis la construction de réponses nouvelles, de la persistance de compétences archaïques avec la réutilisation de solutions toutes faites. Un autre aspect qui est à souligner dans ce processus est constitué par le temps que prennent la construction et la stabilisation de réponses nouvelles : on ne peut pas espérer des changements durables sans environ dix heures de travail effectif des élèves (Marle \& Gréhaigne, 2011). On définira alors le comportement intelligent d'un élève par sa capacité à assurer trois familles articulées de fonctions. Des fonctions de résolution pour élaborer et sélectionner des réponses pertinentes en vue de résoudre les problèmes formulés. Des fonctions de compréhension afin de répondre de façon cohérente et appropriée aux questions qui lui sont adressées et, plus généralement, concevoir et créer des actions en projet signifiantes compréhensibles. Pour un joueur de sports collectifs, nous ajouterons des fonctions d'effectuation, car celles-ci sont organiquement liées aux prises de décision. En effet, à quoi servirait de choisir une réponse qui permettrait de résoudre une tâche partielle de jeu si les compétences motrices nécessaires à son exécution ne sont pas présentes dans le répertoire de réponses disponibles du sujet. 


\section{Apprécier le rapport des forces}

Un joueur de sport collectif constitue un système complexe, où de nombreux éléments en interaction composent différents niveaux d'organisation, avec des états d'équilibre et de déséquilibre variés. Cet ensemble dépend tout d'abord de la façon dont le joueur vit le rapport de forces, l'opposition avec les adversaires et le rapport de compétences à l'intérieur de l'équipe. Les réponses apportées sont aussi dans les caractéristiques de la situation, les contraintes et les opportunités telles que la personne les perçoit.

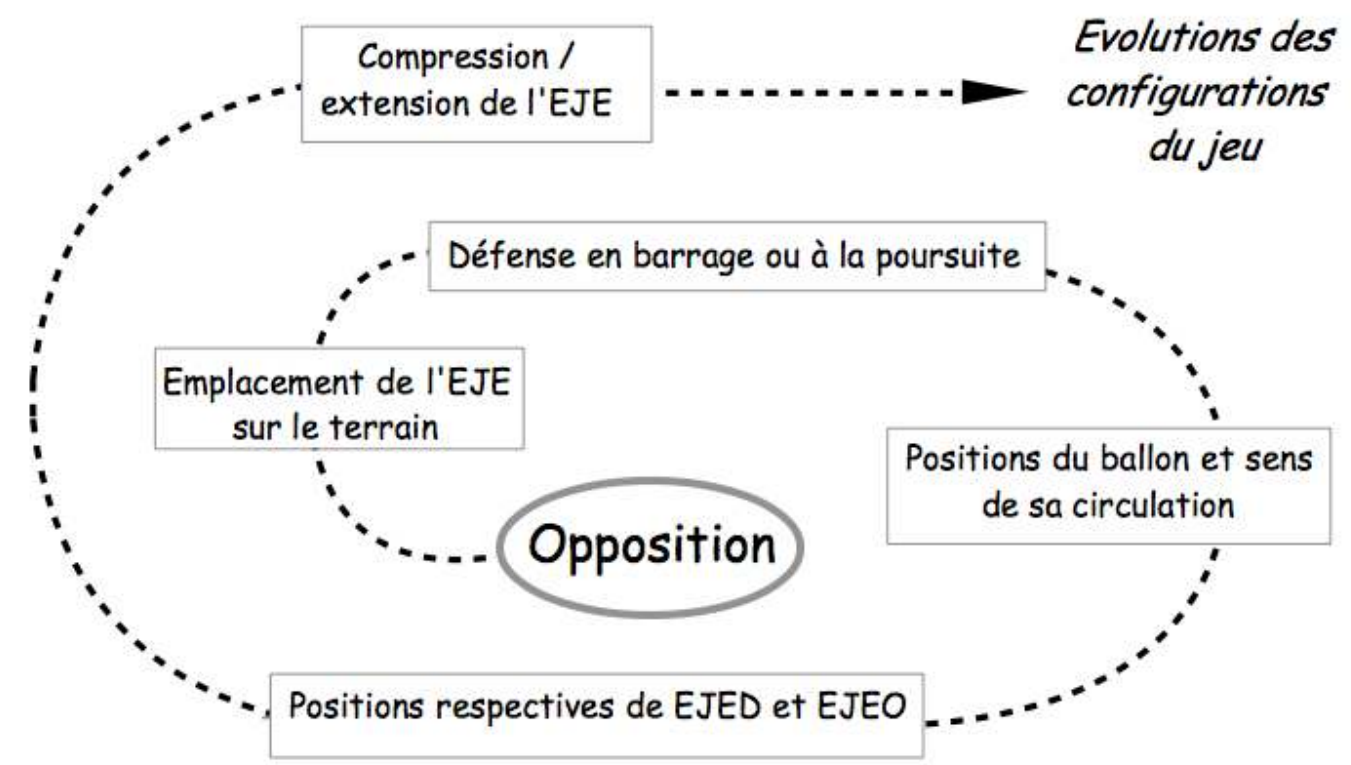

Figure 2. Système de variables pour analyser une configuration momentanée du jeu.

Le modèle de fonctionnement, de développement et d'évaluation du joueur de sport collectif qui vient d'être esquissé met l'accent sur la pluralité des processus et la nature des relations qu'elles entretiennent dans le fonctionnement du joueur. Cette vision semble universelle dans la mesure où le répertoire des processus est le même pour tous les sujets. Elle est différentielle dans la mesure où la hiérarchie entre les éléments du système de variables est changeante selon l'affrontement, les équipes et / ou selon les individus. La figure 2 propose un exemple d'un système de variables pour analyser une configuration momentanée du jeu (Gréhaigne \& Dietsch, 2015).

Ce système de variables forme un ensemble en interaction. Les questions successives permettent de préciser l'état des forces en présence et les rapports d'opposition particuliers.

Ainsi, l'évolution de la dynamique du jeu ne peut alors se modéliser qu'en concevant une évolution discontinue dans le temps. Il nous semble qu'une base de données doit être constituée par la mise en relation de cinq critères :

1) l'emplacement sur le terrain et le volume de l'espace de jeu effectif (l'EJE) ; 
2) la défense en barrage ou à la poursuite ;

3) la position et le sens de la circulation du ballon ;

4) les positions respectives de EJEO et EJED ;

5) l'extension ou la compression de l'EJE en fonction des mouvements du jeu.

Ces critères d'analyse permettent d'obtenir une représentation immédiate et assez exacte du rapport
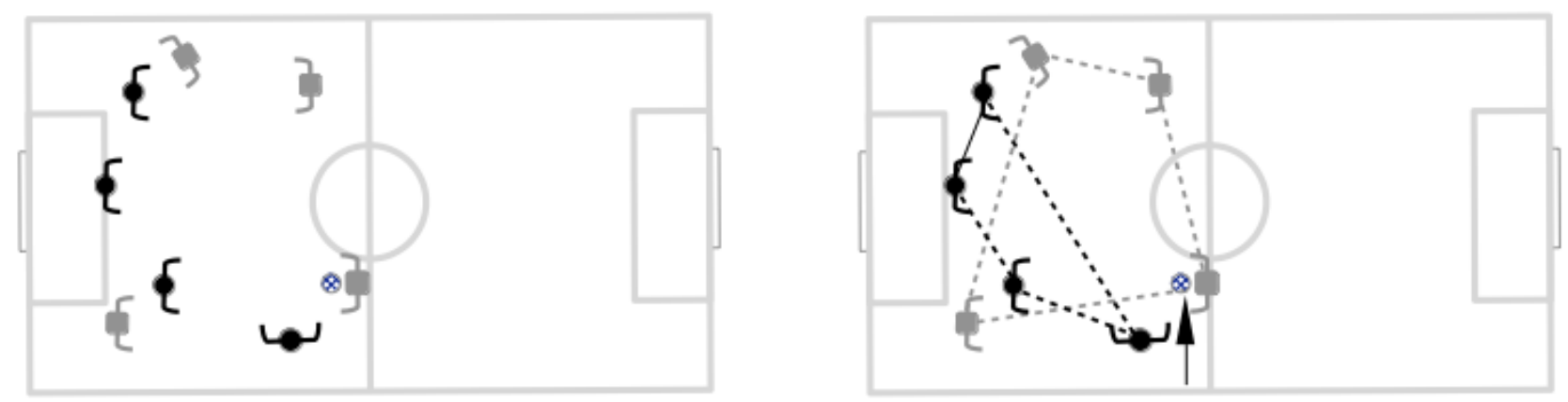

d'opposition en cours. La figure 3 est un exemple pour illustrer l'utilisation de ces critères.

Figure 3. Exemple d'une situation et des EJE des équipes.

Dans la figure 3, l'espace de jeu effectif (EJE) est dans le demi-terrain d'attaque et le ballon est à l'arrière de l'espace de jeu effectif. La défense des ronds noirs est en barrage. Nous sommes donc en présence d'une attaque de position. Dans le cas d'une séquence vidéo, le cinquième critère peut être analysé en fonction des déplacements et formes de l'espace du jeu effectif de l'attaque et de celui de la défense. Nous possédons maintenant une description affinée du point de départ de la situation en vue de mettre en perspective des évolutions. Dans cette situation, le problème à résoudre consiste à forcer ou déplacer le bloc défensif afin de pouvoir s'approcher du but pour tirer. Mérand (1990) propose que face à ce type de configuration, on organise une circulation du ballon et des joueurs.

- soit une circulation du ballon et / ou des joueurs pour mettre en place une configuration opportune qui peut amener une rupture momentanée de l'état d'équilibre du système attaque / défense, avec un danger de but si l'exécution est rapide et bien assurée ; enfin, en cas d'échec de l'action vers le but, si la conservation du ballon est assurée, il est nécessaire d'aller vers d'autres configurations du jeu pour attendre ou provoquer d'autres opportunités ;

- soit une rupture définitive de l'action de jeu par perte de la balle ; les défenseurs récupèrent la balle et deviennent attaquants. En fonction du lieu de récupération, des séquences de jeu (contreattaque, passage rapide, attaque de position) s'imposent pour espérer réussir un but.

Dans le cas de séquences vidéo, il faut se centrer sur l'activité des élèves car les prises de vues devront permettre de concevoir et de construire des situations d'apprentissage adaptées aux 
différentes catégories de réponses repérables. Ce sont donc bien des conduites d'élèves (conduites typiques) qu'il faut repérer de telle façon qu'elles puissent être analysées. On doit aussi noter que, dans cet exemple de situation, en cas de perte de balle par le joueur en possession du ballon, la défense aura beaucoup de mal à revenir en bonne position pour s'opposer à une contre-attaque.

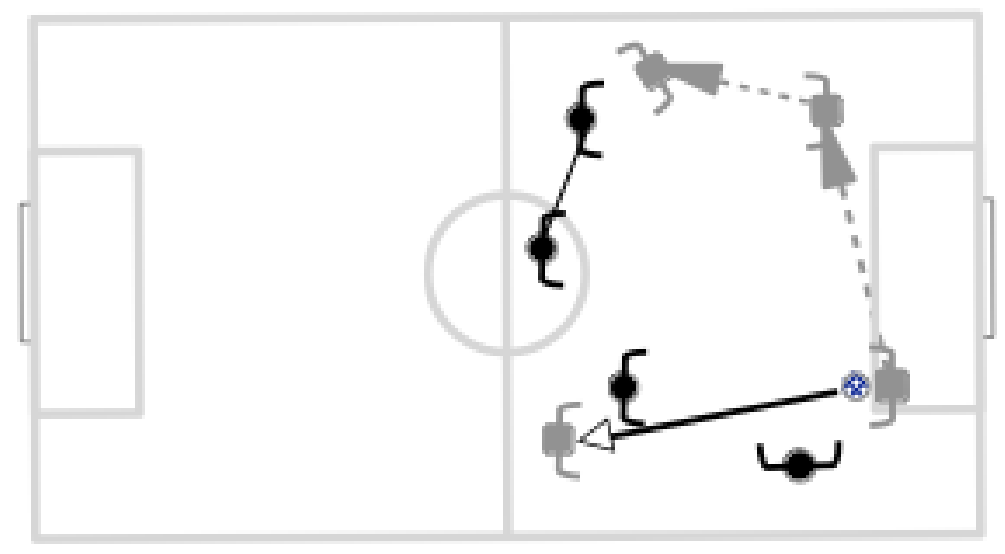

Figure 4. Configuration du jeu identique mais dans un demi-terrain défensif.

La figure 4 montre la même configuration du jeu, mais dans le demi-terrain défensif. On est alors confronté à d'autres problèmes avec la nécessité d'un jeu de transition et d'une montée de la balle pour se rapprocher du but adverse. Le grand coup de pied à suivre est à proscrire car il est très difficile de récupérer la balle qui fuit devant les joueurs. La montée de balle peut être tentée avec un jeu long pour amener le ballon en avant de l'EJE illustrée par la flèche noire, soit avec un jeu en passes courtes en essayant d'éviter de perdre la balle.

\section{Envisager la réversibilité}

Dans le jeu en mouvement, les équipes sont confrontées à l'immédiateté du passage du rôle d'attaquant à celui de défenseur, mettant ainsi en évidence la notion de situation à double effet (Deleplace, 1979). Cette réversibilité des situations représente un aspect fondamental des sports collectifs en rapport avec le fait que les équipes attaquent et défendent tour à tour, et qu'il y a toujours une part de défense dans l'attaque et une part d'attaque dans la défense. On peut ainsi parler d'une véritable «co-construction » du système attaque / défense. Ce système se fait et se défait au gré de l'action. Ce type d'organisation de l'équipe vise, quand on est en défense, à lancer la contre-attaque dès la récupération du ballon à l'aide des joueurs placés en appui. Si l'on attaque, les joueurs en soutien doivent penser qu'ils constituent la première défense contre la contre-attaque en cas de perte du ballon et s'y préparer en conséquence. 
La transition dans la situation à double effet (Deleplace, 1966 ; 1979) est souvent un moment clé, en particulier lors de la récupération du ballon. Dans cette phase, l'ancienne attaque devenue défense doit se réorganiser

- soit s'opposer au front du ballon pour tenter de récupérer vite la balle ;

- soit effectuer un recul fuite pour reconstituer les rideaux défensifs ;

- soit si les rideaux sont en place s'opposer aux pénétrations de l'adversaire.

Pour la défense devenue attaque, on vise :

- soit une circulation du ballon et des joueurs pour mettre en place une contre-attaque avec peu de temps et peu d'échanges pour réussir ;

- soit en fonction de l'état d'équilibre du système attaque / défense, une rupture momentanée de cet équilibre pour amener un but si l'exécution est rapide ;

- soit, en cas d'échec de l'action vers le but et si la conservation du ballon est assurée, poursuivre les actions vers d'autres configurations en vue de faire émerger d'autres opportunités.

A partir du jeu en 7 x 7, la « situation à double effet » repose sur l'existence d'une réserve axiale dynamique et bien en place pour attaquer tout de suite ou être en mesure de s'opposer immédiatement à l'offensive des adversaires en fonction de la possession ou non du ballon. (Marle, Gréhaigne, \& Caty, 2007).

La réversibilité du comportement des joueurs est aussi un autre problème à considérer. En effet, dans des rapports d'opposition, l'embarras avec les connaissances et les compétences motrices découle du fait qu'elles sont susceptibles de dégradation avec la fatigue, le stress, une baisse d'attention voire d'une domination sans partage des adversaires. Cet état d'équilibre est précaire et sa principale caractéristique est la réversibilité, car un joueur, en fonction d'un rapport de forces vécu défavorablement, peut revenir à des conduites relevant d'une adaptation à l'affrontement inférieure à son niveau théorique actuel. Mais si le trouble cesse, l'opposition fléchit ou les causes du désordre prennent fin, le ou les joueurs en reviennent un état d'équilibre antérieur.

Ces phénomènes constituent fréquemment un obstacle à toute évaluation ponctuelle ou partielle des comportements en jeu, car une dose importante d'incertitude subsiste toujours à propos de la stabilité des réponses des joueurs.

\section{Conclusion}

Pour l'étude du système complexe que représente le jeu, l'opposition, la continuité et la réversibilité sont trois concepts qui s'avèrent essentiels. L'opposition renvoie à l'affrontement des deux groupes dans un système dynamique spatialement défini par une aire de jeu et un temps limité dans un match. La continuité du jeu et le jeu en mouvement engendrent du désordre provoquant des désorganisations qui tendent à être compensées tant que la défense peut s'adapter. Bien souvent, ce 
désordre est un désordre où l'on a toutes les raisons de supposer qu'il existe une forme d'ordre caché à qui sait le décoder. Enfin, la réversibilité se produit quand la perturbation n'a pas pu être régulée par les attaquants, entraînant la perte de la balle et le recours ou non à l'organisation à double effet. Les différentes situations d'apprentissage qui amènent à gérer différents types de configurations dans un sport collectif donné sont caractérisées :

- par une aire de jeu avec un espace orienté ;

- un lancement de jeu précis ;

- un équilibre ou un déséquilibre numérique entre les adversaires engagés dans la situation ;

- la réversibilité du jeu doit pouvoir se produire à tout moment, elle est constitutive de la situation ;

- une contrainte temporelle réelle qui pèse sur les élèves.

L'appropriation des connaissances et des compétences par les élèves suppose une évolution des différents concepts, une intégration progressive, par étape à la démarche de pensée qui se matérialise par des niveaux successifs de formulation et d'exploitation (Giordian \& De Vecchi, 1987). Nous ne dirons jamais assez que ces situations d'apprentissage demandent du temps pour qu'elles puissent donner leur plein rendement. Alors soyons patients !

\section{Bibliographie}

Cardinet, J. (1984). Pour apprécier le travail des élèves. Neuchâtel : IRDP.

Deleplace, R. (1966) Le rugby. Paris : Colin-Bourrelier.

Deleplace, R. (1979) Rugby de mouvement - Rugby total. Paris : Éducation Physique et Sports.

Deriaz, D. Poussin, B., \& Gréhaigne, J-F. (1998) Le débat d'idées. Revue EP\&S, 273, 80-82.

Giordan, A., \& De Vecchi, G. (1987). Les origines du savoir. Neuchâtel : Delachaux \& Niestlé.

Gréhaigne, J.F.(2003). Vers une didactique constructiviste en sport collectif. In C. Amade-Escot, (Ed.), Didactique de l'éducation physique : États des recherches (pp. 79-102). Paris : Revue EPS.

Gréhaigne, J.-F., \& Dietsch (2015). L'opposition et ses concepts associés en sports collectifs. In J.F. Gréhaigne, \& G. Dietsch, Quelques aspects théoriques de la didactique des sports collectifs (pp. 88-104). eJRIEPS, Hors Série $\mathrm{N}^{\circ} 1$.

Marle, P., Gréhaigne, J.-F., Caty, D. (2007). Pour une pédagogie des sports collectifs à l'école. In J.-F. Gréhaigne, J.-F. (Ed.) Configurations du jeu, débat d'idées et apprentissage des sports collectifs (pp. 21-42). Besançon : Presses de l’Université de Franche-Comté. 
Marle, P., \& Gréhaigne, J.-F. (2011). Construire des signes et donner du sens aux sports collectifs à l'école. In J.F. Gréhaigne (Ed.), Des signes au sens. Le jeu, les indices, les postures et les apprentissages dans les sports collectifs à l'école (pp. 205-228). Besançon: Presses de l’Université de Franche-Comté.

Mérand, R. (1977). Considérations sur une problématique de rénovation des contenus de l'Education Physique en rapport avec les activités sportives contemporaines. In CPS de la FSGT (Ed.) L'éducateur face à la haute performance olympique (pp.05-27). Paris : Sport et Plein air.

Mérand, R.(Ed.) (1990). Basket-ball : lancer ou circuler ? Rencontres pédagogiques, 28, 9-109. 